\title{
Chronic Groin Pain After Open, Transabdominal Preperitoneal and Totally Extraperitoneal Hernia Repair
}

\author{
Markus Schäfer
}

Published online: 4 February 2010

(C) Société Internationale de Chirurgie 2010

The advent of endoscopic surgery, developments of mesh technology, and increasing cost containment have dominated the debate about inguinal hernia repair during the last decade and a half. In addition, socioeconomic issues (i.e., time to return to work, quality of life) and chronic postoperative pain are being scrutinized.

It has been estimated that $5-10 \%$ of patients after inguinal hernia repair suffer from chronic pain that severely impairs daily life $[1,2]$. Whereas inflammatory and nociceptive types of pain are rather acute, neuropathic pain represents the typical persistent pain following surgery. From a pathophysiologic point of view, nerve damage is an obligatory prerequisite for the development of neuropathic chronic pain [2]. The definition of neuropathic pain includes several criteria that must be fulfilled (Table 1) [3]. A key feature of neuropathic pain is the combination of sensory loss with paradoxical hypersensitivity. Notably, hypoalgesia and hypoesthesia are common sensory disturbances in many patients who have undergone inguinal hernia repair, but its occurrence may be unrelated to chronic pain. There is some evidence that chronic pain "burns out" in half of the patients within 5 years of the operation [4].

Taking a close look at the current literature dealing with chronic pain after inguinal hernia repair, one encounters a bundle of problems and unsolved issues that impairs nearly any meaningful conclusion [2]. Unclear definitions, different methods of pain assessment, lack of long-term

\footnotetext{
M. Schäfer ( $\square)$

Department of Visceral Surgery, Centre Hospitalier

Universitaire Vaudois, University Hospital, Rue du Bugnon 21,

Lausanne 1011, Switzerland

e-mail: markus.schafer@chuv.ch;

markus.schaefer@access.unizh.ch
}

follow-up, and improper study design represent the major shortcomings. In addition, patient-related and surgeryrelated factors must be separately analyzed to differentiate correctly the various aspects of chronic pain. Surgical research has traditionally focused on surgery-related factors (e.g., mesh type, mesh fixation, nerve handling, surgical approaches). Patient-related factors have only rarely been investigated. Nevertheless, it is known that preoperative inguinal pain, young age, pain at other sites of the body, psychosocial factors, and intensity of acute (early postoperative) pain have a major impact on chronic pain [5]. There is also recent evidence that genetic variations may exert major influence on the susceptibility among individuals [2].

A typical example of the surgical research revealing all the methodological difficulties is provided in this issue of the Journal by Bright and colleagues, who report their series of different types of hernia repair with a particular focus on chronic pain [6]. The authors made considerable efforts to analyze their large experience with hernia surgery to gain detailed insight into the frustrating problem of chronic inguinal pain. Unfortunately, the findings are rather disappointing and of limited value. Why?

Table 1 Diagnosis of definite neuropathic pain (all factors must be present)

Pain in a neuroanatomically defined area corresponding to a peripheral or central innervation territory

History of a relevant lesion in the nervous system that is temporally related to development of pain

Partial or complete sensory loss in all or part of the painful area with paradoxical hypersensitivity

Confirmation of a nerve lesion by a specific test (surgical evidence, imaging, clinical neurophysiology, biopsy) 
First, a clear definition of chronic pain is lacking. As a consequence, attendance in the pain clinic after herniorraphy had to be used as a substitute for the incidence of chronic pain. Preoperative inguinal pain was not assessed, and no information is given on early postoperative pain and its analgesic treatment. Second, the diagnostic algorithm and methods to assess patients with chronic pain prior to treatment are not mentioned. Of note, "pain doctors" did not even use a quantitative pain scoring system, either for diagnostic assessment or for treatment control. Thus, how could the diagnosis of chronic inguinal pain and a treatment rationale be established? Third, important technical aspects of the hernia surgery (i.e., the type of mesh and fixation method, nerve handling) are not completely provided. The human factor (i.e., the individual surgical experience) has not been taken into account despite the fact that there were at least 38 consultant teams involved in the hernia surgery. Fourth, a retrospective study design is not appropriate for assessing postoperative chronic pain.

To improve the quality and reliability of future studies, the Initiative on Methods, Measurement, and Pain Assessment in Clinical Trials (IMMPACT) has recommended that six core outcome domains be considered when designing chronic pain clinical trials: pain, physical functioning, emotional functioning, participant ratings of improvement and satisfaction with treatment, symptoms and adverse events, and participant disposition [7]. Such standardized outcome measurement in combination with preoperative pain assessment used in a prospective study design would enable surgeons to obtain evidence-based information on the important issue of chronic inguinal pain concerning its best assessment and treatment, respectively. Furthermore, only multicenter studies using the same protocols can provide adequate patient numbers.

In conclusion, there is increasing knowledge on chronic postoperative pain formation. The surgical community must become aware of those findings to manage patients with chronic pain successfully after inguinal hernia repair.

\section{References}

1. Poobalan AS, Bruce J, Smith WC et al (2003) A review of chronic pain after inguinal herniorrhaphy. Clin J Pain 19:48-54

2. Kehlet H, Jensen TS, Woolf CJ (2006) Persistent postsurgical pain: risk factors and prevention. Lancet 367:1618-1625

3. Rasmussen PV, Sindrup SH, Jensen TS et al (2004) Symptoms and signs in patients with suspected neuropathic pain. Pain 110:461469

4. Kehlet H (2008) Chronic pain after groin hernia repair. Br J Surg 95:135-136

5. Kalkman CJ, Visser K, Moen J et al (2003) Preoperative prediction of severe postoperative pain. Pain 105:415-423

6. Bright E, Reddy MV, Wallace D et al (2010) The incidence and success of treatment for severe chronic groin pain after open, transabdominal preperitoneal, and totally extraperitoneal hernia repair. World J Surg. doi:10.1007/s00268-010-0410-y

7. Dworkin RH, Turk DC, Farrar JT et al (2005) Core outcome measures for chronic pain clinical trials: IMMPACT recommendations. Pain 113:9-19 